\title{
Fluorescence Lifetime Imaging of Coral Fluorescent Proteins
}

\author{
Guy Cox and Anya Salih
}

Electron Microscope Unit, University of Sydney, NSW 2006, Australia

Corals, like many other coelenterates, contain fluorescent pigments which show considerable homology with the well known Green Fluorescent Protein of the jellyfish Aequoria [1]. In corals, unlike jellyfish, multiple proteins are present and the range of excitations and emissions suggest the possibility of energy transfer [2]. The occurrence of FRET between fluorescent proteins in corals has already been reported and timeresolved spectra have shown the effect on fluorescent lifetime, but without any spatial resolution [3]. Lifetime confocal microscopy offers lower time resolution but excellent spatial resolution This study set out to determine the extent of FRET between pigments in two corals which differ in the arrangement of their pigments and hence possibly in pigment function.

Living zooxanthellae and polyp tissue from aquarium-grown Euphyllia ancora and RNAlater ${ }^{\circledR}$ (Ambion Inc, Austin, Texas USA) treated Acropora millepora were examined in seawater. Purified, cloned FPs from A. millepora were provided by M. Matz, University of Florida and were examined either in solution or dried down on a slide. Samples were examined with a Nikon $\mathrm{Cl}$ confocal microscope attached to a Nikon TE2000 inverted microscope and equipped with a Nikon (Europe) LIMO lifetime imaging attachment [4]. Some samples were also examined in a Leica TCS SP2 AOBS confocal microscope fitted with a Becker \& Hickl lifetime imaging module. In both cases picosecond pulsed excitation at $405 \mathrm{~nm}$ was used. For conventional confocal imaging excitation was by $488 \mathrm{~nm}$ argon ion, $561 \mathrm{~nm}$ DPSS and $433 \mathrm{~nm}$ HeNe CW lasers (Fig. 1).

A. millepora has four fluorescent pigments which have been isolated and cloned, and measured lifetimes of the three tested, amilFP490, amilFP504 and amilFP593 (names indicate emission peaks), were 2.8ns, 2.9ns and 2.9ns respectively. These figures lie within the typical range for the GFP family of proteins. In tissue extracted from $A$ millepora, imaging the entire emission spectrum from $420 \mathrm{~nm}$, the mean lifetime was reduced to $1.5 \mathrm{~ns}$, implying that FRET was occurring. Looking just at the fluorescence in the green region (thus likely FRET donors) the lifetime was even shorter, at 1.3ns, supporting this interpretation(Fig. 2). The effect of such a FRET chain is to dump light in a spectral region not usable in photosynthesis, and thus provide photoprotection for the algal symbionts [2].

The pigments of E. ancora have not been cloned but emission spectra taken on the Leica SP2 showed that at least 3 pigments are present, with emission maxima at 506nm, 514nm and 546nm. All pigment granules showed at least two of these, and some clearly contained all three. Yet in fresh Euphyllia tissue the fluorescence lifetime was $2.4 \mathrm{~ns}$ in all regions, suggesting that little or no FRET was taking place (Fig. 3).

The most probable explanation lies in the difference in pigment distribution between the two species. $A$. millepora has uniformly distributed pigment, as would be expected from a photoprotective system. $E$. ancora, on the other hand has a quite striking pattern of bright yellow tips on purple tentacles. This has no correlation with symbiont distribution, and so its purpose, while obscure, is unlikely to be photoprotection. [5] 


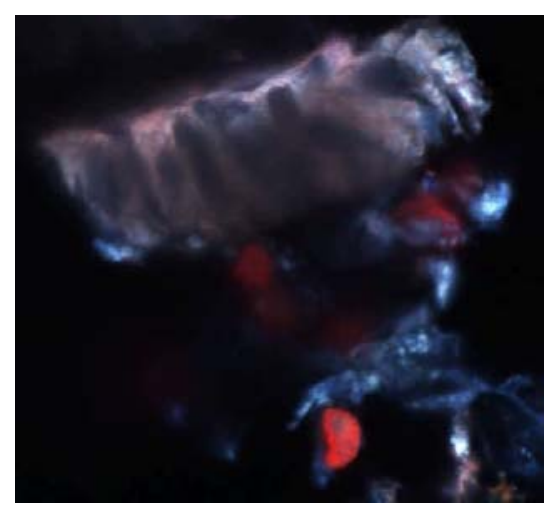

FIG 1. 3-channel confocal fluorescence image of an entire piece of A. millepora in a live-cell chamber. Algal symbionts appear red. a

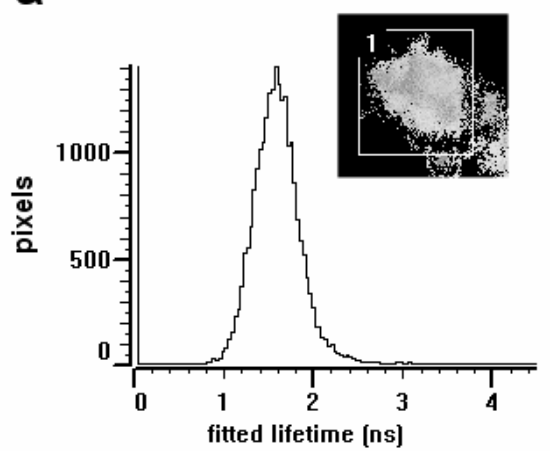

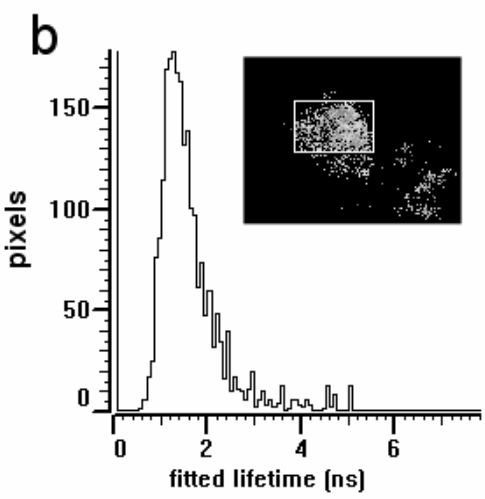

FIG 2. Lifetimes of $A$ millepora fluorescence; (a) in the entire visible spectrum from $420 \mathrm{~nm}$ (modal lifetime $1.56 \mathrm{~ns}$ ) and (b) in the green channel, 515-545 nm (modal lifetime 1.23ns). Nikon LIMO system

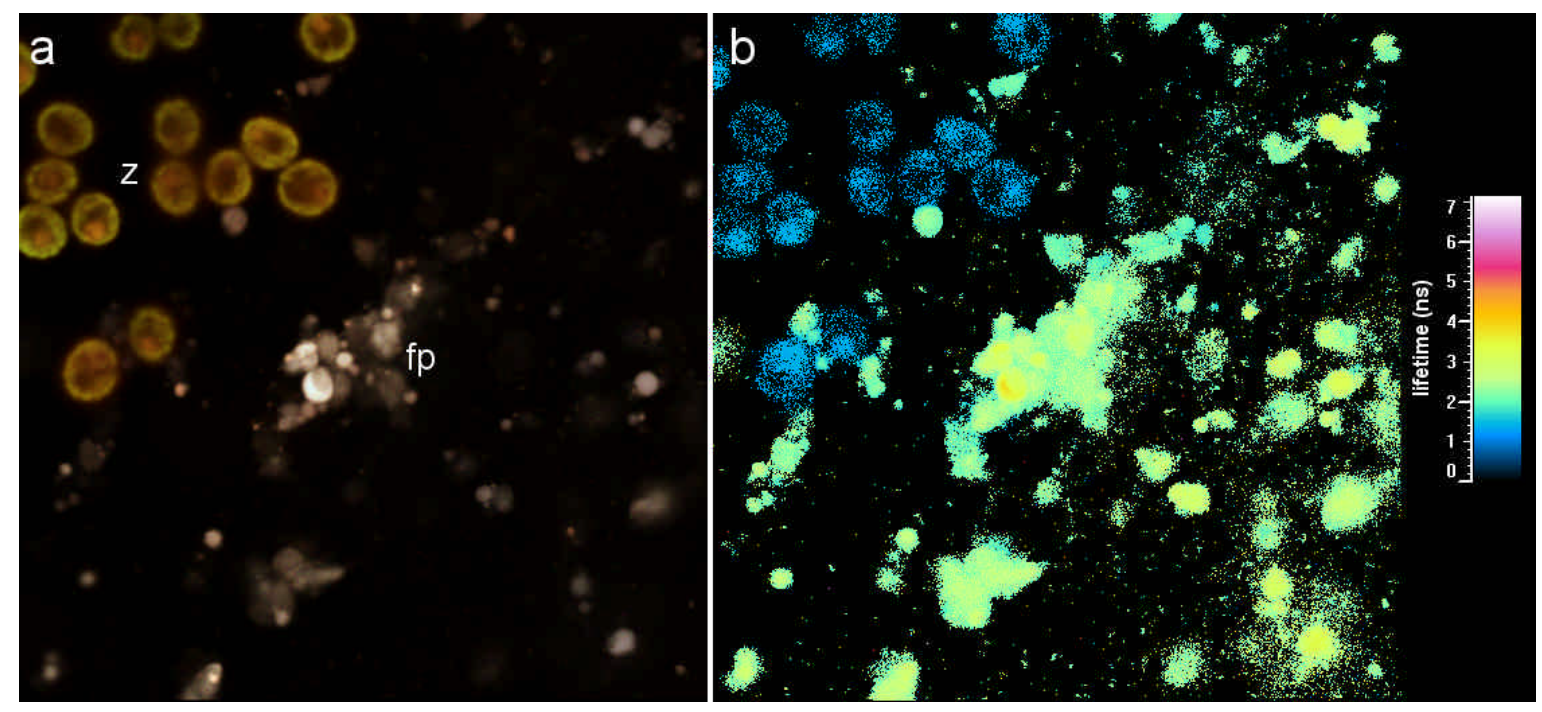

FIG3. E. ancora. (a) lifetime + greyscale image - grey values are true intensity while colour shows lifetime with shortest lifetimes red and longest blue. (b) Lifetime image. $\mathrm{z}$-zooxanthellae, fp-coral fluorescent pigment granules.

\section{References}

[1] M.V Matz et al. Nature Biotech. 17, (1999) 969

[2] A. Salih, A.W.D. Larkum, G. Cox and O. Hoegh-Guldberg. Nature, 408, (2000) 850

[3] A. M.Gilmore, A. W.D. Larkum, Anya Salih, S. Itoh, Y. Shibata, C. Bena, H. Yamasaki, M. Papina and R. Van Woesik. Photochemistry and Photobiology, 77, (2003) 515

[4] M. Balzar. G.I.T. Imaging \& Microscopy 3/2002, (2002) 2

[5] We are very grateful to M. Matz, University of Florida, for the cloned A. millepora pigments. This reseach was supported by Australian Research Council grant DP0451629 and by a University of Sydney Sesqui Fellowship. 